\title{
'
}

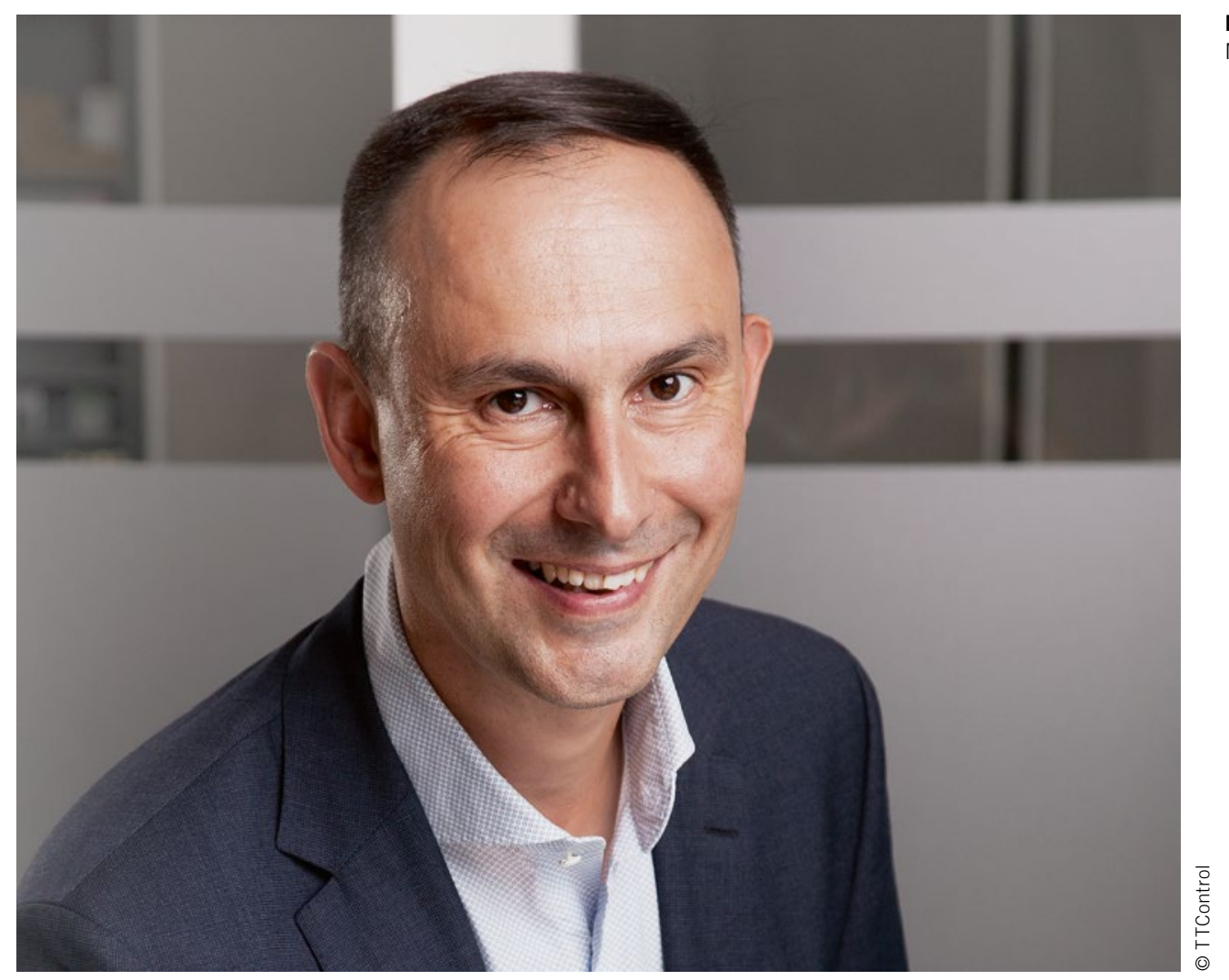

Manfred Prammer

Managing Director TTControl

\section{Certification Saves Cost}

Many mobile machinery builders lose market share due to products that lack safety certifications as the complex certification process is too time-consuming and expensive. It is recommended that OEMs rethink their strategy in this regard as safety certified mobile machinery is regularly being considered in tenders with good cause. Safety certification also reduces liability risks and recourses claims against the manufacturer in the event of damage. One of the most cost-effective ways for a successful certification is using safety-certified components. Safety is about reducing risks to acceptable levels. To achieve this goal, safety standards require products to be developed and produced according to certain processes. These processes are complex and time-consuming. As a part of these processes, safety risks must be identified, and specific requirements must be established to address those risks. To fulfill these requirements, safety-related functionalities or mechanisms must be in place. They are usually allocated to some components in the vehicle architecture, e.g. Electronic Control Units (ECUs). Today's ECUs are quite powerful. They can perform various monitoring and diagnostic functions. On the occurrence of a safety-critical error, an ECU enters the safe state. More specifically, safety-critical outputs are switched off.
Such diagnostic and error-response mechanisms implemented in ECUs are rather complex technical topics. Their design and implementation require highly qualified experts that are both scarce and costly. By using certified components, economies of scale can be achieved, and valuable qualified human resources can be conserved, which means that common complex problems are solved by a specialized supplier once for many customers. OEMs still have a job to do. Of course, certified components like ECUs cannot guarantee the standard compliance of the final control system implemented on top of the platform. Since an OEM's application running on the ECU is not known in the first place, a definitive safety function can only be assumed. The ISO 26262 standard defines such systems as Safety Element out of Context (SEooC). As there is no "one size fits all” electronic component, each OEM must still weigh the pros and cons, based on their specific conditions and needs. Overall, the usage of certified electronic components is very beneficial as it reduces the scope of the certification of systems and time to market is reduced by having complex problems already solved in a ready-to-use component. 